Results 28 interviews were conducted (15 MSMO, 13 MSMW). Half of MSMW reported predominantly male partners and half predominantly female. MSMO viewed STIs as "an annoying reality of life" and both groups had positive perceptions of STI testing; however, MSMW described more STI stigma and less frequent testing. MSMO and MSMW who were more involved with the queer community had better sexual health knowledge. Many MSMW noted it was easier to have sexual health discussions with male partners; however, many also described the pressure of condom use, "with men, [was] to not use a condom a fair bit of time and probably by women, [was] to use a condom." There was significant fear in both groups about disclosing sexual practices to general practitioners and some MSMW preferred the anonymity of specialist sexual health clinics. Biphobia and bisexual erasure were frequently discussed by both groups.

Conclusion MSMW described less comprehensive sexual health knowledge and more barriers to accessing sexual health care. Service provision and health promotion messaging must be broadened to capture the reality of increasing sexual fluidity. Destigmatising MSM behaviour and sexual health discussions, particularly in primary care, is crucial to ensuring all people receive appropriate sexual health care.

Disclosure No significant relationships.

\section{P519 IS CHEMSEX AMONG MEN WHO HAVE SEX WITH MEN PERCEIVED AS PROBLEMATIC? A CROSS-SECTIONAL STUDY IN THE NETHERLANDS}

\begin{abstract}
${ }^{1}$ Ymke Evers, ${ }^{1}$ Christian Hoebe*, ${ }^{1}$ Nicole Dukers-Muijers, ${ }^{2}$ Karlijn Kampman, ${ }^{3}$ Sophie Kuizenga, ${ }^{4}$ Decontee Shilue, ${ }^{5}$ Nienke Bakker, ${ }^{6}$ Sophie Schamp, ${ }^{1}$ Geneviève Van Liere. 'Public Health Service South Limburg, Maastricht University Medical Center (MUMC), Sexual Health, Infectious Diseases and Environmental Health, Medical Microbiology, Care and Public Health Research Institute (CAPHRI), Heerlen, Netherlands; ${ }^{2}$ Public Health Service Twente, Sexual Health, Twente, Netherlands; ${ }^{3}$ Public Health Service Haaglanden, Sexual Health, Den Haag, Netherlands; ${ }^{4}$ Public Health Service Middle Brabant, Sexual Health, Middle Brabant, Netherlands; ${ }^{5}$ Public Health Service Middle Gelderland, Sexual Health, Middle Gelderland, Netherlands; ${ }^{6}$ Public Health Service Brabant South-East, Sexual Health, Brabant South-East, Netherlands
\end{abstract}

10.1136/sextrans-2019-sti.597

Background Men who have sex with men (MSM) are increasingly using drugs during sex ('chemsex') and this has been associated with several health harms, including an increased risk for sexually transmitted infections (STI) and addiction. Little evidence exists on whether chemsex is perceived as problematic by MSM. This study assessed a wide range of social and behavioral aspects in MSM engaging in chemsex.

Methods In 2018, 785 MSM were recruited at eight Dutch STI clinics, of which $511(65 \%)$ completed the online questionnaire. Chemsex was defined as using cocaine, crystal meth, designer drugs, GHB/GBL, ketamine, speed or XTC/MDMA during sex in the preceding six months. Characteristics of MSM intending to change chemsex behaviour and MSM with a need for professional counselling were described using $\chi^{2}$ tests.

Results Chemsex was reported by 41\% (209/511). Among MSM engaging in chemsex, intention to change was reported by $19 \%$ (40/209); decreasing the frequency of drug use was mostly reported $(70 \%(28 / 40))$. Intention to change was highest among MSM who had no sex without drugs $<3$ months $(44 \%(12 / 47)$ vs $15 \%, \mathrm{p}<0.001)$, reported unwanted sexual experiences $(36 \%(14 / 39)$ vs $15 \%, \quad \mathrm{p}=0.01)$, and used $\geq 5$ drugs $<6$ months $(31 \%(16 / 51)$ vs $16 \%, \mathrm{p}=0.03)$. The need for professional counselling was reported by $23 \%$ (48/209). The majority wanted to be counselled on increasing self-control $(52 \%(25 / 48))$. The need for professional counselling was highest among MSM who had an intention to change $(45 \%$ $(18 / 40)$ vs $18 \%, p<0.001)$, had no sex without drugs $<3$ months $(41 \%(11 / 27)$ vs $20 \%, p=0.04)$, and engaged in chemsex $\geq 2$ times per month $(30 \%(28 / 93)$ vs $17 \%, \mathrm{p}=0.03)$.

Conclusion Our study shows that one in five MSM engaging in chemsex reported an intention to change or expressed a need for professional counselling. STI healthcare providers should discuss chemsex frequency, multiple drug use, sober sex and unwanted sexual experiences, and if necessary refer to professional addiction-or mental healthcare.

Disclosure No significant relationships.

\section{P520 HPV INFECTIONS AND FLAT PENILE LESIONS OF THE PENIS IN MEN WHO HAVE SEX WITH MEN}

${ }^{1}$ Ward Van Bilsen*, ${ }^{1}$ Alexandra Kovaleva, ${ }^{2}$ Maaike Bleeker, ${ }^{3}$ Audrey King, ${ }^{1}$ Sylvia Bruisten, ${ }^{4}$ Wilma Brokking, ${ }^{5}$ Henry De Vries, ${ }^{2}$ Chris Meijer, ${ }^{1}$ Maarten Schim Van Der Loeff*. ${ }^{1}$ Public Health Service of Amsterdam, Department of Infectious Diseases, Amsterdam, Netherlands: ${ }^{2}$ Amsterdam UMC, Vrije Universiteit-University Medical Center, Department of Pathology, Amsterdam, Netherlands; ${ }^{3}$ Center for Infectious Disease Control, National Institute for Public Health and the Environment (RIVM), Bilthoven, Netherlands; ${ }^{4}$ DC Klinieken, Department of Internal Medicine, Amsterdam, Netherlands; ${ }^{5}$ Public Health Service Amsterdam, Amsterdam University Medical Center (UMC), National Institute of Public Health and the Environment (RIVM), Infectious Diseases, Infection and Immunity Institute (AI and II), Epidemiology and Surveillance Unit, Amsterdam, Netherlands

\subsection{6/sextrans-2019-sti.598}

Background Flat penile lesions (FPL) in heterosexual men are thought to play a role in the transmission of HPV. We investigated the association between FPL and penile HPV, and explored determinants of FPL in men who have sex with men (MSM).

Methods In 2015-2016, MSM were recruited based on HIV and penile HPV status in a previous study. MSM self-completed a questionnaire. Peniscopy was performed after application of acetic acid to visualize FPL. Penile physician-collected samples were tested for HPV-DNA using the highly sensitive SPF10-PCR DEIA/LiPA25 system. If tested positive for HPV $6,11,16,18,31,33,35,39,45,51,52,56,58$ and/or 59, we determined the HPV viral load (VL), using type-specific L1-targeting quantitative (q)PCR. Presence of HPV and HIV, HPV VL and circumcision status were compared between MSM with and without FPL.

Results We included 116 MSM, of whom 59/116 (51\%) were HIV-positive and 54/116 (47\%) had FPL. A penile HPV infection was present in 31/54 (57\%) MSM with FPL and in 34/ $62(55 \%)$ MSM without FPL $(\mathrm{p}=0.8)$. Among MSM with FPL, 16/54 (30\%) had an hrHPV infection and 23/54 (43\%) had a lrHPV infection, which did not significantly differ from MSM without FPL $(p=0.5$ and $p=0.4$, respectively). A detectable HPV VL was found in 10/54 (19\%) MSM with FPL and in 10/62 $(16 \%)$ MSM without FPL $(p=0.6)$. Among MSM with FPL, $27 / 54$ (50\%) were HIV-positive and 5/54 (9\%) were circumcised, and among MSM without FPL, 32/62 (54\%) were HIV-positive and 13/62 (21\%) were circumcised $(\mathrm{p}=0.9$ and $\mathrm{p}=0.09$, respectively).

Conclusion Among MSM in Amsterdam, we found no association between FPL and penile HPV, HPV VL, HIV status or circumcision status, which is in contrast with findings among 
heterosexual males. Our findings imply that FPL are not useful in identifying HPV infections with a high transmission potential in this population.

Disclosure No significant relationships.

\section{P521 INCREASES IN THE ESTIMATED NUMBER OF REPORTED GONORRHEA CASES AMONG MEN WHO HAVE SEX WITH MEN (MSM): THE ROLE OF TESTING}

${ }^{1}$ Austin Williams, ${ }^{1}$ Emily Weston*, ${ }^{2}$ Thomas Gift, ${ }^{1}$ Elizabeth Torrone. ${ }^{1}$ US Centers for Disease Control and Prevention, Division of STD Prevention, Atlanta, USA; ${ }^{2}$ Centers for Disease Control and Prevention, Division of STD Prevention, Atlanta, USA

\subsection{6/sextrans-2019-sti.599}

Background Interpreting trends in rates of reported cases of gonorrhea is difficult because infections, particularly extra-genital infections, are often asymptomatic and increased screening coverage will result in increased case detection. In the absence of national data on screening coverage among men who have sex with men (MSM), we estimated trends in MSM testing in the United States given a range of positivity estimates.

Methods We estimated the number of tests that would have had to occur to identify the estimated number of reported gonorrhea cases among MSM in a given year. The number of tests was calculated by dividing the annual number of estimated cases among MSM (based on sentinel surveillance) by estimated test positivity among MSM (based on published literature). We calculated the estimated number of tests under both low positivity (3.5\%) and high positivity (10\%), as well as the effect of stable and changing positivity during 20162017.

Results The estimated number of reported gonorrhea cases among MSM increased by 17.9\% during 2016-2017 (from 163,537 to 192,740 ). We estimated that 1.9 million (assuming $10 \%$ positivity) to 5.5 million (assuming $3.5 \%$ positivity) tests performed among MSM would be needed to detect the estimated number of gonorrhea cases among MSM in 2017. This represents an increase of approximately 290,000 to 830,000 tests over the estimated number of tests conducted in 2016. Alternatively, if the number of tests was stable over time, a 0.6 to 1.8 percentage point increase in positivity from 2016 to 2017 would be consistent with the increase in observed cases.

Conclusion We provide a framework to inform trends in case rates by analyzing the impact of changes in positivity and testing over time. Our analysis implies that estimated increases in reported gonorrhea cases among MSM likely resulted from both increased screening and increased incidence.

Disclosure No significant relationships.

\section{P522 DISPARITIES IN HIVISTI TESTING AND DIAGNOSIS AMONG URBAN AND NON-URBAN US MEN WHO HAVE SEX WITH MEN FROM 2013 TO 2017}

Maria Zlotorzynska*, Travis Sanchez, Patrick Sullivan. Emory University Rollins School of Public Health, Department of Epidemiology, Atlanta, USA

\subsection{6/sextrans-2019-sti.600}

Background Most data about HIV/STI testing and diagnosis among US men who have sex with men (MSM) comes from urban areas, though the majority of the population resides outside of these areas. Since 2013, the American Men's Internet Survey (AMIS) has conducted annual nationwide online behavioral surveillance of $\geq 10,000$ US MSM.

Methods Participants age $15+$ were recruited through online advertisements. County urban/rural categories were based on National Center for Health Statistics classification. Poisson models using generalized estimating equations tested associations between urban/rural category and HIV testing, STI (syphilis, gonorrhea, chlamydia) testing and STI diagnoses in the past 12 months. All models controlled for survey year, age, race/ethnicity, insurance, HIV status (except model for HIV testing) and recruitment source.

Results From 2013 through 2017, 49,903 completed surveys were collected: $42.4 \%$ MSM from urban counties, $20.5 \%$ suburban, $28.3 \%$ small/medium metro, and $8.8 \%$ rural. STI testing was more prevalent in urban counties (50.2\%) compared to suburban $(37.8 \%, p<0.0001)$, small/medium metro $(35.6 \%, p<0.0001)$ and rural $(27.8 \%, p<0.0001)$ counties. STI diagnoses were more prevalent in urban counties (13.4\%) compared to suburban $(8.1 \%, p<0.0001)$, small/ medium metro $7.5 \%, p<0.0001)$ and rural $(5.4 \%$, $p<0.0001)$ counties. Among HIV-negative/unknown status MSM, HIV testing was more prevalent among MSM from urban counties (61.9\%) compared to suburban $(52.3 \%$, $p<0.0001)$, small/medium metro $(50.6 \%, p<0.0001)$ and rural $(43.6 \%, p<0.0001)$ counties. Significant trends over time were observed in HIV testing for all counties, while STI testing only increased in urban and small/medium metro counties. STI diagnoses increased significantly in all but rural counties.

Conclusion Urban/rural disparities in HIV/STI testing and STI diagnoses were found in a multi-year national sample of US MSM. These findings likely reflect disparate geographical distribution of healthcare access and resources. If these disparities cannot be adequately addressed in programs that reach underserved areas, nationwide HIV/STI prevention goals for MSM will not likely be met.

Disclosure No significant relationships.

\section{P523 URETHRAL MICROBIOTA IN IDIOPATHIC NON- GONOCOCCAL URETHRITIS (NGU) IN MEN WHO HAVE SEX WITH MEN AND MEN WHO HAVE SEX WITH WOMEN}

${ }^{1}$ Sujatha Srinivasan*, ${ }^{2}$ Laura Chambers, ${ }^{3}$ Ken Tapia, ${ }^{4}$ Noah Hoffman, ${ }^{1}$ Matthew Munch, ${ }^{5}$ Jennifer Morgan, ${ }^{1}$ Daniel Domogala, ${ }^{6} \mathrm{M}$ Lowens, ${ }^{4}$ Sean Proll, ${ }^{7} \mathrm{M}$ Huang, ${ }^{1}$ Keith Jerome, ${ }^{4}$ Matthew Golden, ${ }^{8}$ James Hughes, 'David Fredricks, ${ }^{2}$ Lisa Manhart. ${ }^{1}$ Fred Hutchinson Cancer Research Center, Vaccine and Infectious Disease Division, Seattle, USA; ${ }^{2}$ University of Washington, Epidemiology, Seattle, USA; ${ }^{3}$ University of Washington, Global Health, Seattle, USA; ${ }^{4}$ University of Washington, Medicine, Seattle, USA; ${ }^{5}$ Public Health - Seattle and King County, Seattle, USA; ${ }^{6}$ Public Health - Seattle and King County, HIVISTD Program, Seattle, USA; ' University of Washington, Laboratory Medicine, Seattle, USA; ${ }^{8}$ University of Washington, Biostatistics, Seattle, USA

10.1136/sextrans-2019-sti.601

Background NGU is common with no known etiology in $\sim 50 \%$ of cases. We evaluated the association of urethral bacteria with NGU among men who have sex with men (MSM) and men who have sex with women (MSW).

Methods Urine samples were collected from MSM and MSW attending a Seattle STD Clinic and enrolled in a cross-sectional study. Chlamydia trachomatis (CT) and Mycoplasma genitalium (MG) were detected by TMA (Aptima), and adenovirus, HSV-1 and HSV-2 by PCR. NGU was defined as having urethral 